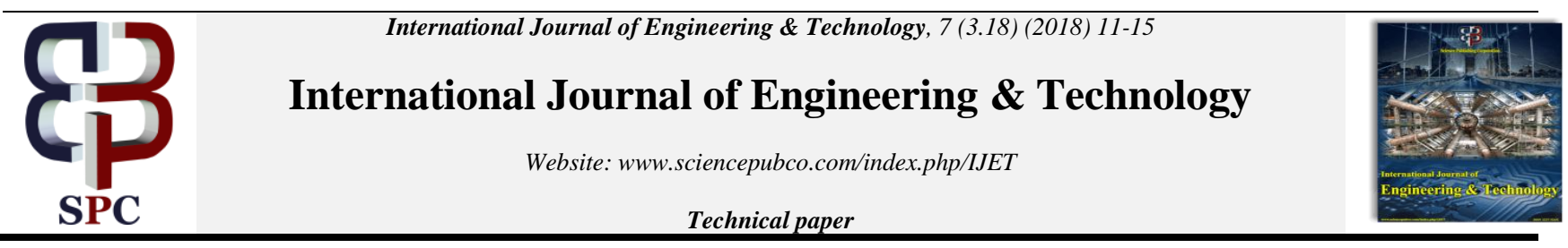

\title{
Development of Automatic Solar Tracking System for Small Solar Energy System
}

\author{
Musse Mohamud Ahmed ${ }^{1 *}$, Mohammad Kamrul Hasan ${ }^{1}$, and Mohammad Shafiq ${ }^{1}$ \\ ${ }^{1}$ Department of Electrical and Electronics Engineering, Faculty of Engineering, Universiti Malaysia Sarawak. \\ *Corresponding author E-mail: mamusse@unimas.my
}

\begin{abstract}
The main purpose of this paper is to present a novel idea that is based on design and development of an automatic solar tracker system that tracks the Sun's energy for maximum energy output achievement. In this paper, a novel automatic solar tracking system has been developed for small-scale solar energy system. The hardware part and programming part have been concurrently developed in order for the solar tracking system to be possible for it to operate accurately. Arduino Uno R3, Sensor Shield V4 Digital Analog Module, LDR (Light Dependent Resistor), MPU-6050 6DOF 3 Axis Gyroscope has been used for tracking the angular sun movement as shown in Fig. 1. Accelerometer, High-Efficiency Solar Panel, and Tower Pro MG90S Servo Motor have been used for the hardware part. High-level programming language has been embedded in the hardware to operate the tracking system effectively. The tracking system has shown significant improvement of energy delivery to solar panel comparing to the conventional method. All the results will be shown in the full paper. There are three contributions the research presented in this paper which are, i.e. perfect tracking system, the comparison between the static and tracking system and the development of Gyroscope angular movement system which tracks the angular movement of the sun along with another tracking system.
\end{abstract}

Keywords: MPU-6050 6DOF; Solar Panel, Solar Tracker.

\section{Introduction}

Over the recent years, the foremost reasons behind global warming are greenhouse effects, which have caused precarious climate changes all over the world. Unfortunately, these severe weather changes are the negative effects of damaging the Earth. Many methods and attempts have been used to tackle the continual global warming in order to reduce its effect. For instance, the Kyoto Protocol, which has been signed and endorsed by many national governments in an effort to educate people and correspondingly improve their environmental awareness about limited current energy sources and their relatively high demand. The main purpose of this study is to design and develop an automatic solar tracker system. At the present, the use of solar energy is not new. The known drawback of widely used solar panels is not maximizing its utilization to fully absorb the solar energy. It is believed that the sunlight has two basic components. These are the "direct beam" that contains about $90 \%$ of the solar energy and the "diffuse sunlight" that carries the remaining solar energy. Therefore, the aim of this project is to improve on the function of the regular solar panel with an addition of LDRs, motor, gear and wheel mounting.

Malaysia is situated in the North of the Equator where the exact latitude and longitude are $2^{\circ} 30^{\prime} \mathrm{N}$ and $112^{\circ} 30^{\prime} \mathrm{E}$ respectively. At this location, solar irradiation can be optimally extracted. Contrary to the usage of non-renewable energies such as coal, gasoline, and oil, solar power has become more popular as an environmentally friendly renewable energy source that produces no pollution and requires minimal maintenance. Moreover, energy from the Sun is free and also has an advantage of reducing power usage from conventional energy resources based on fossil fuel. It is, therefore, necessary to maximize the solar energy usage through solar panels and conversion system. Hence, one of the means to maximize solar energy power delivery is to use the solar tracker. The Gyroscope angle tracking system shown in Fig. 1 has perfectly tracked the angular movement of the sun and strengths of the tracking system.

This also shows the movements of the motor and angular movement showing that what angle each rotation stands for the solar tracking positions.

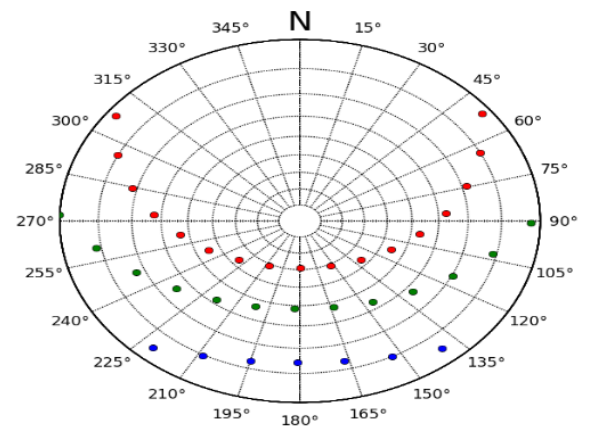

Fig. 1: Gyroscope Angle Tracking 